\title{
ECG Morphological Decomposition for Automatic Rhythm Identification
}

\author{
Guadalupe García-Isla ${ }^{1}$, Rita Laureanti ${ }^{1}$, Valentina D. Corino ${ }^{1}$, Luca T. Mainardi ${ }^{1}$ \\ ${ }^{1}$ Department of Electronics, Information and Bioengineering, Politecnico di Milano, Milan, Italy
}

\begin{abstract}
Manual rhythm classification in 12-lead ECGs is timeconsuming and operator-biased. We present an automatic ECG classifier using CinC's 2020 challenge dataset. In the first phase of the Challenge, 9 categories were targeted with an ensemble of 4 classifiers. In the second phase, 7 classifiers were implemented to detect 24 cardiac electrophysiological disorders. Five classifiers identified abnormalities in different specific regions of the heart's conducting system. Two classifiers were dedicated to detect premature atrial and ventricular contractions.

The methodology is based on the creation of rhythmspecific intra and inter-patient templates. Firstly, signals were divided into 6 regions of interests. Secondly, for each region, intra-patient models and inter-patient rhythmspecific models were computed. The distances from each intra-patient model to each rhythm-specific inter-patient model as well as heart rate variability features and Global Electric Heterogeneity features were introduced into the classifiers.

After a 10-fold cross-validation, for the provided training data in the first phase an accuracy of $94.4 \% \pm 0.4$, and a Challenge metric of $0.644 \pm 0.031$ were obtained, whereas in the second phase an accuracy and Challenge metric of $15.0 \pm 1.0 \%$ and $0.030 \pm 0.009$ were obtained.
\end{abstract}

\section{Introduction}

Cardiovascular diseases (CVDs) are the first cause of mortality and morbidity worldwide [1]. The standard 12lead electrocardiogram (ECG) is an essential tool in clinical practice to diagnose CVDs and to have an initial assessment of a patient's health condition [2]. Typically, manual interpretation of ECGs by expert clinicians is needed. This requires skilled personnel with high degree of specialization and in some cases inter-operators discrepancies are present. An automatic algorithm for detection of cardiac abnormalities and classification of ECG recordings could aid clinical practice, providing clinicians with an objective tool to make an early and accurate diagnosis of CVDs [3]. The aim of this work is to put together machine learning techniques and physiological know-how to build and validate such model, using the 6 annotated datasets available for the 2020 Computing in Cardiology Challenge [4] (team name: Germinating).

\section{Material and Methods}

\subsection{Data}

Six databases were provided: China Physiological Signal Challenge in 2018 (CPSC2018), St Petersburg INCART 12-lead Arrhythmia Database, the PTB Diagnostic ECG Database, the PTB-XL electrocardiography Database and the Georgia 12-Lead ECG Challenge Database. In total, 43,101 labeled 12-lead ECGs with 111 different cardiac abnormalities labelled following the SNOMED-CT coding system. Only 24 disorders were targeted: 1st degree AV block (IAVB), atrial fibrillation (AF), atrial flutter (AFL), bradycardia (Brady), incomplete right bundle branch block (IRBBB), left anterior fascicular block (LAnFB), left axis deviation (LAD), left bundle branch block (LFBBB), low QRS voltages (LQRSV), nonspecific intraventricular conduction disorder (NSIVCB), pacing rhythm (PR), premature atrial complex (PAC), premature ventricular complex (PVC), prolonged PR interval (LPR), prolonged QT interval (LQT), Q-wave abnormal (QAb), right axis deviation (RAD), sinus arrhythmia (SA), sinus bradycardia (SB), normal sinus rhythm (NSR), sinus tachycardia (STach), T-wave abnormal (TAb) and T-wave inversion (TInv), the rest were considered "Unscored" and ignored. For the first phase, also ST elevation (STE) and depression (STD) were considered.

\subsection{ECG preprocessing}

Two median filters were applied to obtain the baseline of each ECG signal that was then subtracted to the original ECG to obtain a baseline corrected signal. Power line and high-frequency noise were removed with a finite impulse response low-pass filter with equal ripple in the pass and stop bands. The 3-dB point of the filter was $35 \mathrm{~Hz}$. R peaks were detected using Pan Tompkins' algorithm [5]. To minimize misdetections, $\mathrm{R}$ peaks were detected on 5 leads with positive QRS (I, II, III, aVF, V4, V5, V6). An impulse 
train signal was built based on the $\mathrm{R}$ peaks detected for each lead; a Gaussian filter was applied to smooth the $\mathrm{R}$ series, then signals were aligned through a crosscorrelation procedure, using V5 as reference and finally the median signal among the 5 series was computed. On the obtained signal, the Pan Tomkins algorithm was applied to obtain the $\mathrm{R}$ peaks used on the rest of the analysis.

\subsection{Heart rate variability features}

The mean and standard deviation of the RR intervals was computed, along with the standard deviation of the difference of consecutive RR intervals, the percentage of successive interval differences greater than $50 \mathrm{~ms}$ (pNN50) and the root mean square of successive differences (RMMSD).

\subsection{Intra and inter-patient models}

Inter-patient models that gathered the median behaviour of each electrohpysiological disorder in the ECG were built for different signal segments of clinical significance. These models were used to measure the distance of each patient to the ECG pattern typical of each electrophysiological disorder.

Firstly, ECG windows of interest were selected based on the expected occurrence of different electrical events (all values referred to the location of $\mathrm{R}$ peak): $\mathrm{P}$-wave (from -300 ms to $-40 \mathrm{~ms}$ ) [6], QRS-complex (-70 ms to +60 $\mathrm{ms}$ ), PQ-ST (PT-segment with the removal of the QRSsegment, from $-150 \mathrm{~ms}$ to $+250 \mathrm{~ms}$, removing the segment from $-55 \mathrm{~ms}$ to $+55 \mathrm{~ms}$ ), $\mathrm{T}$-wave (from $+100 \mathrm{~ms}$ to +350 $\mathrm{ms}$ ) [7], PR (from $-288 \mathrm{~ms}$ to the R peak), RT (from the R peak to $+258 \mathrm{~ms}$ ).

Secondly, intra-patient templates were computed for each of the above-mentioned segments. For each lead, all windows were aligned and the median waveform, representative of the analyzed subject, was calculated. The mean standard deviation of all windows was stored as a feature. Thirdly, the maximum cross-correlation index was computed between each individual region of interest and the median intra-patient template. Fourthly, for each rhythm, the templates of the correspondent subjects of the training set were aligned and the median rhythm template was obtained. Finally, the maximum cross-correlation between each intra-patient templates and each of the interpatient models with the corresponding lags were stored as features. In Figure 1, an example is visible for the RT segment for NSR, RBBB and LBBB. On the left panels, all RT segments in the recording of a subject are depicted with the obtained intra-patient template in red. On the right, the median intra-patient templates of all the subjects are plotted with the resulting inter-patient template in red.

\subsection{GEH features}

Global Electric Heterogeneity (GEH) features were computed using the three open source toolboxes provided by the challenge: HRV toolbox [8], ECGkit [9] and GEH parameter extraction [10], [11] and origin point [12]. These features included: azimuth, elevation, and magnitude of spatial peak QRS, T and spatial ventricular gradient (SVG) vectors, azimuth and elevation of QRS, T vectors and Wilson's area SVG, scalar value of the SVG, and peak and are of the spatial QRS-T angle [10], [11].

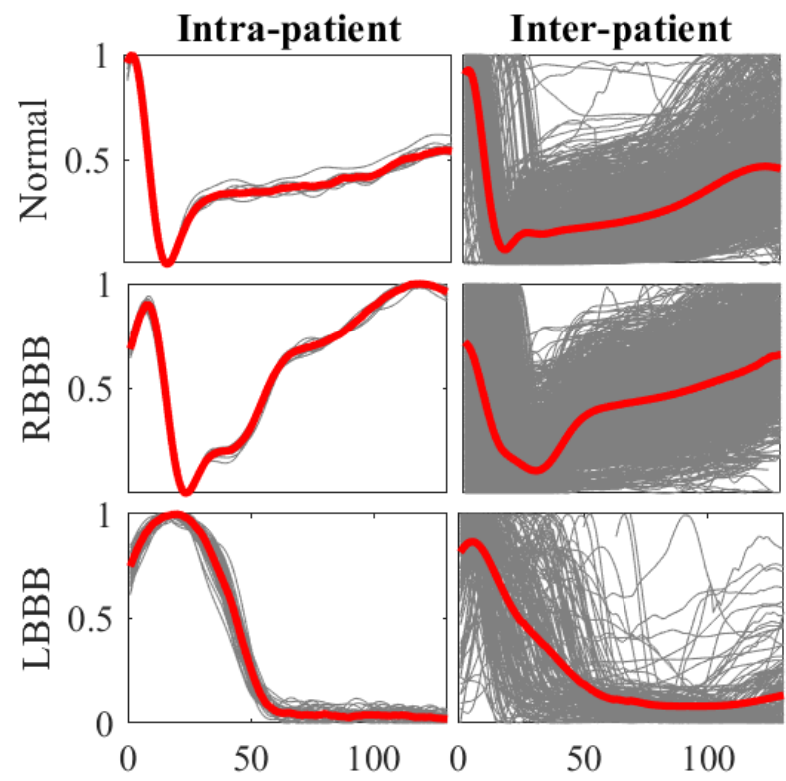

Figure 1. Intra and inter-patient templates for the RT segment of the rhythms Normal, RBBB and $L B B B$. All the segments of a recording are overlapped in the intra-patient plots, whereas in the inter-patients plots, the median segments of all the subjects with the respective condition are depicted. The median segments are in red.

\subsection{Classifier ensemble}

The classification strategy adopted was to create an ensemble of classifiers, each of them focused on a specific region of the cardiac conductive system. A distinction was made between sporadic conditions as PAC and PVC, and stable conditions, all the other pathologies. As most signals ranged from $6 \mathrm{~s}$ to $60 \mathrm{~s}$, all arrhythmias were considered present on the whole signal.

During phase 1 , the detection of permanent conditions was distributed into different classifiers depending on the ECG region where the electrophysiological disorders were manifested (Table 1). This yielded one classifier (C1) including the cross-correlation values between interpatient and intra-patient of the P-wave and PR interval, and another one (C2) including the QRS complex, RT segment and T-wave information. Both classifiers included GEH and HRV features, as well as age and sex.

Given the increase in the amount of rhythms included in phase 2, the detection of permanent conditions was 
distributed differently, according to the cardiac region of occurrence:

- C1: Atrial tissue

- Purkinje system

$$
\begin{array}{ll}
\circ & \text { C2: Sinoatrial node } \\
\circ & \text { C3: Atrioventricular (AV) node } \\
\circ & \text { C4: Bundle branches }
\end{array}
$$

- C5: Ventricular tissue

Cross-correlation values between inter-patient and intra-patient models were included as: P-wave and PR segments for $\mathrm{C} 1$ and $\mathrm{C} 3$, all segments for $\mathrm{C} 2$, and QRS complex, PQ-ST segment, T wave, and RT segment for C4 and C5. HRV and GEH features as well as age and sex were included in all classifiers.

For each of the sporadic conditions (PAC and PVC) an individual classifier was designed in both phases, which led to a total of 4 classifiers in phase 1 and 7 in phase 2. C6 and $\mathrm{C} 7$ included all the HRV features and the crosscorrelation values between the intra-patient template and the 3 beats with the shortest preceding RR interval for the QSR and P-wave segments. Signals classified as AF or AFL were not taken into consideration for possible PAC or PVC.

In phase $1, \mathrm{C} 1$ and $\mathrm{C} 2$ were support vector machines. PAC and PVC classifiers used bagged trees. For the second phase, in which 24 categories were included, all classifiers used boosted trees. All classifiers were implemented in Matlab 2020a and trained using a 10-fold cross-validation.

\section{Results}

The results obtained after the 10 -fold cross-validation of the provided datasets for phase 1 and 2 of the challenge are gathered in Tables 1 and 2, respectively. Sensitivity and specificity values are displayed along with the number of signals present in the dataset for each rhythm of study. In addition, the sub-indexes $\mathrm{C} 1, \mathrm{C} 2, \ldots, \mathrm{C} 7$ in the Rhythms column indicate the classifier in which the rhythm was included. In both phases specificity (Sp.) values were higher than sensitivity (Se.) ones. Results in phase 1 were superior than those in phase 2. Categories not detected by the classifier in phase 2 have been excluded from Table 2.

Table 3 gathers the overall results obtained for the classifier ensembles of phases 1 and 2 after a 10-fold crossvalidation on the databases provided. The Challenge
Metric (CM) used to evaluate the classifiers varied from phase 1 to phase 2 . While in phase 1 it corresponded to the geometrical mean between $F_{\beta}$ measure and $G_{\beta}$ measure, on phase 2 a new scoring system was used to reflect the value of the algorithm in a clinical setting: it awarded full credit to correct diagnoses and partial credit to misdiagnoses with similar risks or outcomes similar to those of the actual diagnosis.

Table 1. Classification results on the test set after 10-fold cross-validation on the CPSC dataset labelled according to the first phase of the challenge.

\begin{tabular}{llll}
\hline Rhythms & Subjects & $\mathrm{Se}(\%)$ & $\mathrm{Sp}(\%)$ \\
\hline $\mathrm{AF}_{\mathrm{C} 1}$ & 1221 & $94.6 \pm 0.02$ & $95.9 \pm 0.01$ \\
IAVB $_{\mathrm{C} 1}$ & 722 & $88.1 \pm 0.05$ & $97.1 \pm 0.01$ \\
LBBB $_{\mathrm{C} 2}$ & 236 & $85.6 \pm 0.08$ & $98.5 \pm 0.00$ \\
NSR & 918 & $78.8 \pm 0.04$ & $93.7 \pm 0.01$ \\
PAC $_{\mathrm{C} 3}$ & 616 & $84.5 \pm 0.06$ & $90.3 \pm 0.01$ \\
PVC $_{\mathrm{C} 4}$ & 700 & $87.8 \pm 0.02$ & $93.5 \pm 0.01$ \\
RBBB $_{\mathrm{C} 2}$ & 1857 & $89.5 \pm 0.02$ & $96.5 \pm 0.01$ \\
STD $_{\mathrm{C} 2}$ & 869 & $69.6 \pm 0.05$ & $96.8 \pm 0.01$ \\
STE $_{\mathrm{C} 2}$ & 220 & $37.2 \pm 0.09$ & $99.3 \pm 0.00$ \\
\hline
\end{tabular}

Table 2. Classification results on the test set after 10-fold cross-validation on all the datasets labelled according to the second phase of the challenge.

\begin{tabular}{llll}
\hline Rhythms & Subjects & $\mathrm{Se}(\%)$ & $\mathrm{Sp}(\%)$ \\
\hline AF $_{\mathrm{C} 1}$ & 3475 & $94.55 \pm 1.19$ & $93.97 \pm 0.56$ \\
AFL $_{\mathrm{C} 1}$ & 314 & $16.33 \pm 6.77$ & $99.70 \pm 0.10$ \\
IAVB $_{\mathrm{C} 3}$ & 2394 & $92.32 \pm 1.82$ & $81.08 \pm 1.76$ \\
IRBBB $_{\mathrm{C} 4}$ & 1611 & $5.78 \pm 2.43$ & $99.77 \pm 0.14$ \\
LAD $_{\mathrm{C} 4}$ & 6086 & $56.52 \pm 5.13$ & $86.84 \pm 0.83$ \\
LAnFB $_{C 4}$ & 1806 & $56.29 \pm 14.67$ & $97.60 \pm 0.58$ \\
LBBB $_{C 4}$ & 1041 & $31.38 \pm 3.64$ & $99.71 \pm 0.09$ \\
LQT $_{C 5}$ & 1513 & $8.91 \pm 2.98$ & $99.08 \pm 0.54$ \\
PAC $_{C 6}$ & 1944 & $65.88 \pm 4.20$ & $91.61 \pm 0.84$ \\
PVC $_{C 7}$ & 1253 & $50.22 \pm 7.17$ & $85.36 \pm 1.45$ \\
RBBB $_{C 4}$ & 3085 & $89.53 \pm 2.39$ & $95.70 \pm 0.52$ \\
SB $_{C 2}$ & 2359 & $43.20 \pm 2.69$ & $98.95 \pm 0.20$ \\
NSR $_{C 2}$ & 20846 & $90.48 \pm 0.61$ & $70.38 \pm 1.55$ \\
STach $_{C 2}$ & 2402 & $86.78 \pm 2.91$ & $99.25 \pm 0.20$ \\
TAb $_{C 5}$ & 4673 & $56.57 \pm 7.34$ & $78.75 \pm 4.07$ \\
\hline
\end{tabular}

Table 3. Classification results on phase 1 and 2 for 10 -fold cross-validation of the provided datasets.

\begin{tabular}{llllllll}
\hline Ph. & AUROC & AUPRC & Accuracy & F & Fbeta & Gbeta & CM \\
\hline $1^{\text {st }}$ & $0.099 \pm 0.009$ & $0.019 \pm 0.002$ & $0.944 \pm 0.004$ & $0.727 \pm 0.019$ & $0.763 \pm 0.019$ & $0.525 \pm 0.025$ & $0.644 \pm 0.031$ \\
$2^{\text {nd }}$ & $0.541 \pm 0.002$ & $0.083 \pm 0.001$ & $0.148 \pm 0.009$ & $0.116 \pm 0.004$ & $0.144 \pm 0.004$ & $0.062 \pm 0.002$ & $0.030 \pm 0.009$ \\
\hline
\end{tabular}

$\mathrm{Ph}=$ phase; AUROC=area under the receiver operating curve; AUPRC=area under the precision-recall curve. 


\section{Discussion}

The aim of this work was to propose an automatic algorithm capable of identifying different cardiovascular diseases using 6 different databases with 43,101 labeled recordings made available by the PhysioNet/Computing in Cardiology Challenge 2020 [4]. Several attempts have been already described in literature [3], [13], [14], [15]. Currently, the role of clinicians is still fundamental for the final diagnosis, but a support role from computers could provide a useful tool to aid them for early and correct diagnosis of cardiac abnormalities.

The presented method intended to follow a physiologically consistent approach. An ensemble of classifiers was built focusing each of them on specific cardiac regions. The electrophysiological disorders of study were distributed into each of the classifiers depending or their region of incidence. ECG signals were divided into regions of interest and a comparison was performed between intra-patient models and inter-patient rhythm-specific models. In phase 1 the methodology succeeded in detecting all disorders with specificity higher than $90 \%$ and sensitivity higher than $84 \%$, except for STE and STD. These results are in line with those already present in literature [15], although as different databases are used, a comparison is not trivial. These results suggest that intra-patient and inter-patient models manage to capture electrophysiological disturbances of different nature and areas of the cardiac tissue.

However, results obtained in phase 2 show that the metrics used are not enough if a larger number of conditions with often similar expression in the ECG are targeted, as IRBBB and RBBB, IAVB and LPR, LAnFB and $\mathrm{LBBB}$, among others. In each classifier $(\mathrm{C} 1, \mathrm{C} 2, \ldots$, C7) at least a cardiac abnormality was detected with good results i.e. $\mathrm{AF}$ in $\mathrm{C} 1$, $\mathrm{STach}$ in $\mathrm{C} 2$, IAVB in $\mathrm{C} 3$, RBBB in C4. However, the models built and/or the metric obtained seemed to be unable to distinguish among such an amount of rhythms exhibiting similar morphologies.

\section{Conclusion}

We obtained promising results for the detection of a limited number of electrophysiological abnormalities in short signals. Further development is needed for its application to a high number of disorders, possibly through the implementation of convolutional neural network.

\section{Acknowledgments}

This project is framed inside MY-ATRIA consortium. MY-ATRIA project has received funding from the European Union's Horizon 2020 research and innovation program under the Marie Skłodowska-Curie grant agreement No.766082.

\section{References}

[1] M. F. Piepoli et al., "2016 European Guidelines on Cardiovascular Disease Prevention in Clinical Practice," Eur. Heart J., vol. 37, no. 29, pp. 2315-2381, 2016.

[2] N. Peters, M. A. Gatzoulis, and R. Vecht, ECG Diagnosis in Clinical Practice, Second. Springer, 2009.

[3] H. Smulyan, "The Computerized ECG: Friend and Foe," Am. J. Med., vol. 132, pp. 153-160, 2019.

[4] E. A. Perez Alday et al., "Classification of 12-lead ECGs: the PhysioNet/Computing in Cardiology Challenge 2020," Physiol. Meas., 2020.

[5] J. Pan and W. J. Tompkins, "A Real-Time QRS Detection Algorithm," IEEE Trans. Biomed. Eng., vol. BME-32, no. 3, pp. 230-236, 1985.

[6] F. Censi et al., "P-Wave Morphology Assessment by a Gaussian Functions-Based Model in Atrial Fibrillation Patients," IEEE Trans. Biomed. Eng., vol. 54, no. 4, pp. 663-672, 2007.

[7] G. Goovaerts, C. Varon, B. Vandenberk, R. Willems, and S. Van Huffel, "Tensor-based Detection of T wave Alternans in Multilead ECG Signals," in Computing in Cardiology, 2014.

[8] A. N. Vest et al., "An Open Source Benchmarked Toolbox for Cardiovascular Waveform and Interval Analysis," Physiol. Meas., vol. 39, no. 10, p. 105004, 2018.

[9] A. J. Demski and M. Llamedo Soria, "ecg-kit a Matlab Toolbox for Cardiovascular Signal Processing," J. Open Res. Softw., vol. 4, no. 1, p. e8, 2016.

[10] J. W. Waks et al., "Global Electric Heterogeneity Risk Score for Prediction of Sudden Cardiac Death in the General Population: The Atherosclerosis Risk in Communities (ARIC) and Cardiovascular Health (CHS) Studies," Circulation, vol. 133, pp. 2222-2234, 2016.

[11] J. A. Thomas et al., "Vectorcardiogram in Athletes: The Sun Valley Ski Study," Ann. Noninvasive Electrocardiol., p. e12614, 2018.

[12] E. A. Perez-Alday et al., "Importance of the Heart Vector Origin Point Definition for an ECG Analysis: The Atherosclerosis Risk in Communities (ARIC) Study," Comput. Biol. Med., vol. 104, pp. 127-138, 2019.

[13] M. Alfaras, M. C. Soriano, and S. Ortín, "A Fast Machine Learning Model for ECG-based Heartbeat Classification and Arrhythmia Detection," Front. Phys., vol. 7, p. 103, 2019.

[14] J. Schläpfer and H. J. Wellens, "Computer-Interpreted Electrocardiograms: Benefits and Limitations," J. Am. Coll. Cardiol., vol. 70, no. 9, pp. 1183-1192, 2017.

[15] X. Zhang et al., "Automated Detection of Cardiovascular Disease by Electrocardiogram Signal Analysis: A Deep Learning System," Cardiovasc. Diagn. Ther., vol. 10, no. 2, pp. 227-235, 2020.

Address for correspondence:

Guadalupe García Isla

Via Camillo Golgi, 39, 20133 Milano, Italy

guadalupe.garcia@polimi.it 